\title{
Self-Consistent Solution of the Multi Band Boltzmann, Poisson and Hole-Continuity Equations
}

\author{
SURINDER P. SINGH*, NEIL GOLDSMAN and ISAAK D. MAYERGOYZ \\ Department of Electrical Engineering, University of Maryland College Park, MD 20742
}

\begin{abstract}
The Boltzmann transport equation (BTE) for multiple bands is solved by the spherical harmonic approach. The distribution function is obtained for energies greater than $3 \mathrm{eV}$. The BTE is solved self consistently with the Poisson equation for a one dimensional $n p n$ bipolar junction transistor (BJT). The novel features are : the use of boundary fitted curvilinear grid, and Scharfetter Gummel type discretization of the BTE.
\end{abstract}

Keywords: Boltzmann Transport Equation, Curvilinear Grid.

\section{INTRODUCTION}

The Monte Carlo (MC) method, the most prominent of the BTE solution techniques, has the disadvantage of being computationally very expensive. The spherical harmonic approach for solving the BTE, which represents a compromise method in between hydrodynamic modeling and full zone MC simulation, is computationally efficient, and therefore has been gaining acceptance [1-3].

This paper presents new extensions to the past work, which includes: (i) incorporation of all bands from the multi-band band structure [4] ; (ii) self consistency with the Poisson and hole-continuity equations ; (iii) a new Scharfetter-Gummel like discretization for the BTE; and (iv) a novel boundary fitted curvilinear grid.

\section{ANALYTICAL MODEL}

Along with the Poisson and the hole-continuity equations the analytical device model consists of the
Boltzmann transport equations (BTE) for each band $v,(v=1,2,3,4)$. The dimensionality of the BTE is reduced by projecting it on a spherical harmonics basis in $\mathbf{k}$ space, $f(\mathbf{r}, \mathbf{k})=\Sigma_{l, m} f_{l}^{m}(\mathbf{r}, k) Y_{l}^{m}(\theta, \phi)$ where, $k=|\mathbf{k}|, \theta$ is the polar angle, and $\phi$ is the azimuthal angle. By substituting the expansion into the BTE, and applying the orthogonality of $\mathrm{Y}_{l}^{m}$ and changing variables $f_{0}{ }^{0}(x, \varepsilon)$ to $F_{0}{ }^{0}(x, H)=f_{0}{ }^{0}(x, H+q \phi)$ we get $[2,3]$ :

$$
\frac{\tilde{v}}{3 \gamma}\left\{\frac{\partial}{\partial x}\left(\tau \tilde{\tilde{r} \gamma} \frac{\partial F_{0}^{0}}{\partial x}\right)\right\}+\left[\frac{\partial F_{0}^{0}}{\partial t}\right]_{\text {coll }}=0
$$

Where $\gamma(\varepsilon)=\hbar^{2} k^{2} / 2 m^{*}$, is the band shape function for each band of the multi-band band structure from Brunetti et. al [4]. The model consists of four bands. For computational simplicity the two lower bands and the two upper bands can be combined together and are enumerated by index $(I)$ and (II). The scattering mechanisms considered are intra-band acoustic phonon scattering in the elastic approximation, intra-band inter-valley optical phonon, inter-band optical phonons, impact ionization.

\footnotetext{
* Corresponding author. Tel: (301) 405-7540. Fax: (301) 314-9281. Email: surinder@eng.umd.edu
} 


\section{DISCRETIZATION ON CURVILINEAR GRID}

The BTE (1) is defined on a curvilinear region bounded by $x=0, L_{x}$ and $H=-q \phi(x), \varepsilon_{\max }-q \phi(x)$. The curvilinear nature of the problem is exploited by resorting to a curvilinear grid which conforms to this boundary and performing interpolations when writing the finite difference approximations of the derivatives [5]. Fig. 1 depicts the schematic of the grid. There are three major advantages of this grid : (i) the grid can be made selectively dense in energy $\varepsilon$; (ii) during self consistent calculations the grid remains the same ; and (iii) the boundary condition at band maxima is directly specifiable.

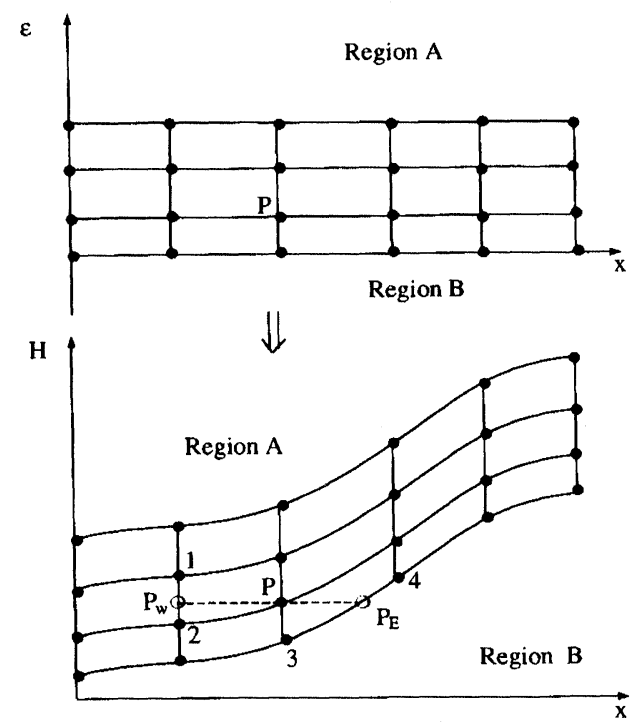

FIGURE 1 The boundary fitted curvilinear grid is first constructed in the $(x, \varepsilon)$ space, and then it is mapped to the $(x, H)$ space (by the relation $H=\varepsilon-q \phi$ ) for actual simulations. Linear interpolation is performed for finite-difference discretizations. During self-consistent calculations when $\phi$ changes from one Gummel iteration to the other, the grid does not change intrinsically, but instead merely readjusts itself to the new $\phi$

The equations are discretized by finite differences and by using Scharfetter-Gummel type discretization of equation (1). The individual equations are solved using a fixed point SOR approach, while the overall system is solved self consistently with a decoupled Gummel-type scheme.

\section{RESULTS}

As shown in Fig. 2 two designs of the BJT are simulated, $n$-type collector and $p$-type collector.

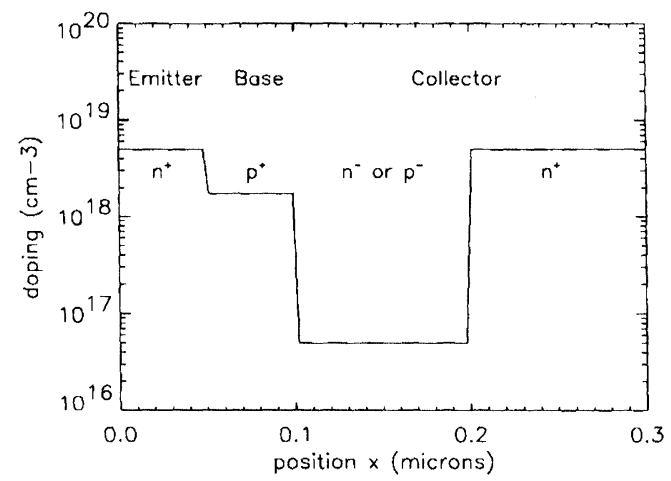

FIGURE 2 The doping profiles for the simulated BJTs. The lightly doped part of the drain is depleted for both $n$ and $p$ design

Fig. 3 shows the self consistent electric fields at the bias of $V_{B E}=0.83 \mathrm{~V}$ and $V_{C E}=2.25 \mathrm{~V}$ for the two collector designs. Fig. 4 displays the distribution function in the $n$-type design. The electron velocity is plotted in Fig. 5. Velocity overshoot is present at the beginning of the collector-base space charge region. Fig. 6 displays the average energy of the electrons. The ionization coefficient, as shown in Fig. 7, is larger for the $p$-type design because of the stronger

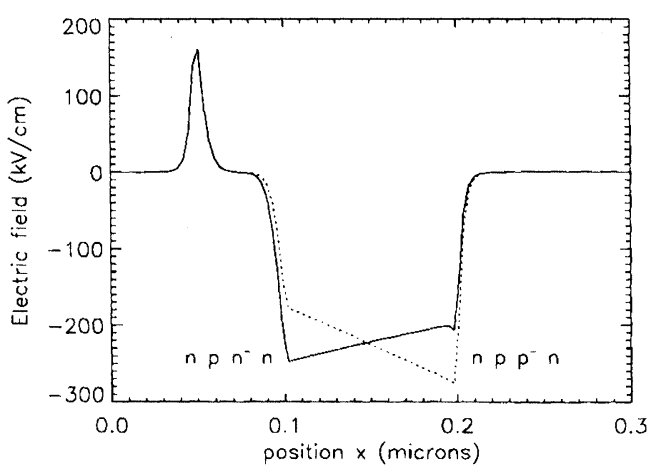

FIGURE 3 The self consistent electric field for the two BJTs 

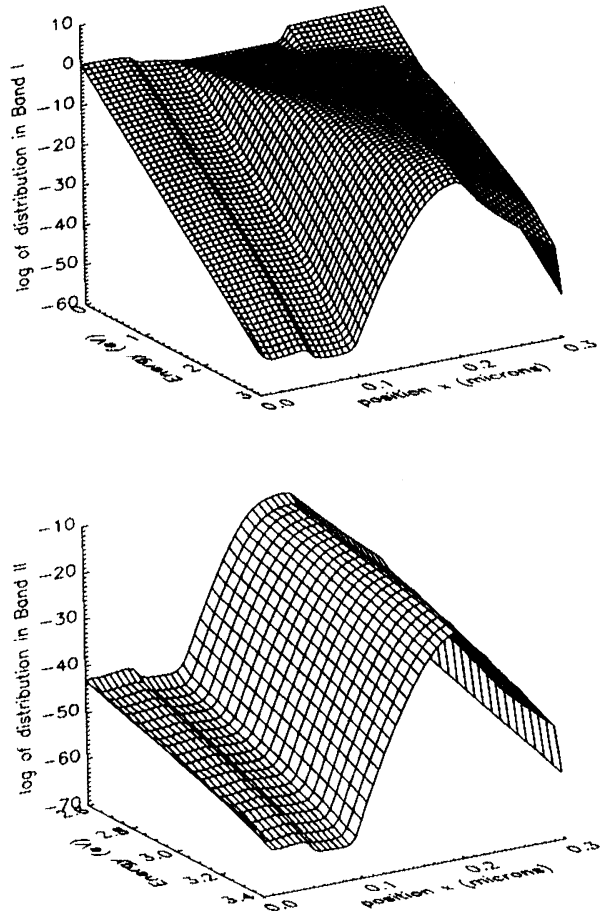

FIGURE 4 Distribution function in the two bands of the multiband band structure for the BJT. Band $(I)$ is the concatenation of the lower bands, and (II) is the concatenation of the upper bands. The electrons heat up as they traverse from the emitter to the collector region. The heated electrons scatter to the upper bands, and the population of band (II) increases by many orders of magnitude

electric field causing a larger heating of the carriers. Impact ionization scattering pushes the distribution towards equilibrium in the neutral collector. Fig. 8 plots the distribution at a location in the collector with and without impact ionization.

\section{CONCLUSIONS}

We have modeled the transport of high-energy electrons in a BJT by a self-consistent solution of the multi-band BTE along with the Poisson and the holecontinuity equation. The BTEs are solved by spherical harmonic expansion approach. The numerical

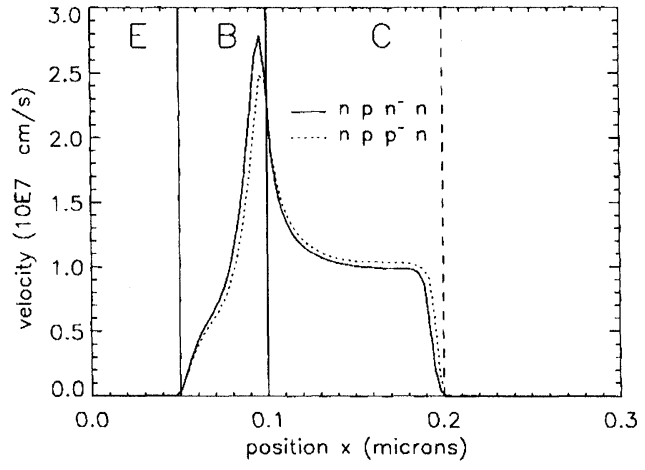

FIGURE 5 The plot of electron velocity as function of position in the $n$-type and $p$-type design

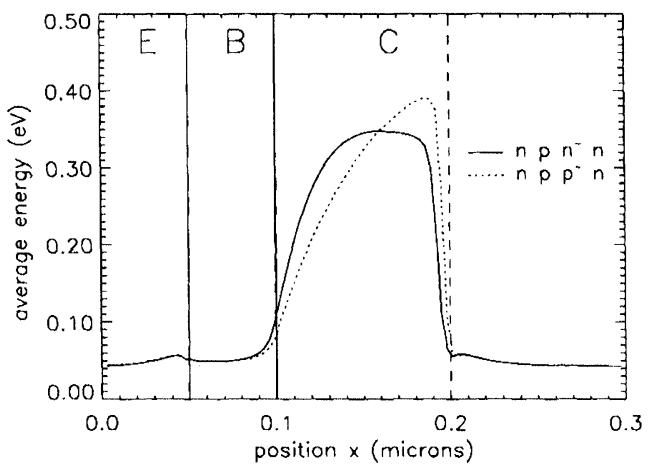

FIGURE 6 The plot of average electron energy as function of position in the $n$-type and $p$-type BJT design

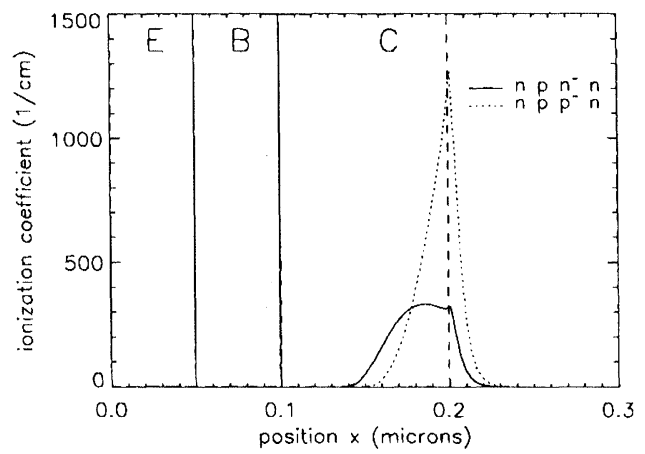

FIGURE 7 The ionization coefficient as function of position in the $n$-type and $p$-type BJT design 
scheme employs a boundary fitted curvilinear grid and performs Scharfetter-Gummel discretization of the resulting BTE.

\section{References}

[1] H. Lin, N. Goldsman, I. D. Mayergoyz, Solid-State Electron., 35(6), 769, 1992.

[2] A. Gnudi, D. Ventura, G. Baccarani, IEEE trans. on CAD, 12(11), 1706, 1993.

[3] K. A. Hennacy, et. al., Y. -J. Wu, N. Goldsman, I. D. Mayergoyz, Solid-State Electron. 38(8), pp. 1485-1495, 1995.

[4] R. Brunetti, C. Jacoboni, F. Venturi, E. Sangiorgi, and B. Ricco, Solid-State Electron., 32(12), 1663, 1989.

[5] J. F. Thompson, Z. U. A. Warsi, J. Comp. Phys., 47, 1, 1982.

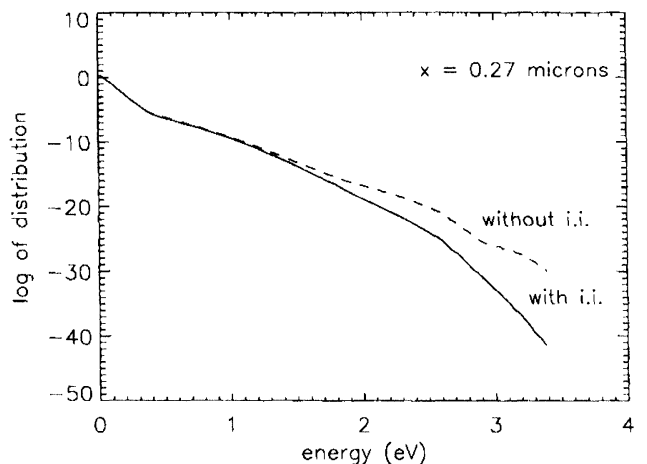

FIGURE 8 Effect of impact ionization in the collector. The prsence of impact ionization tends the distribution towards equilibrium faster 

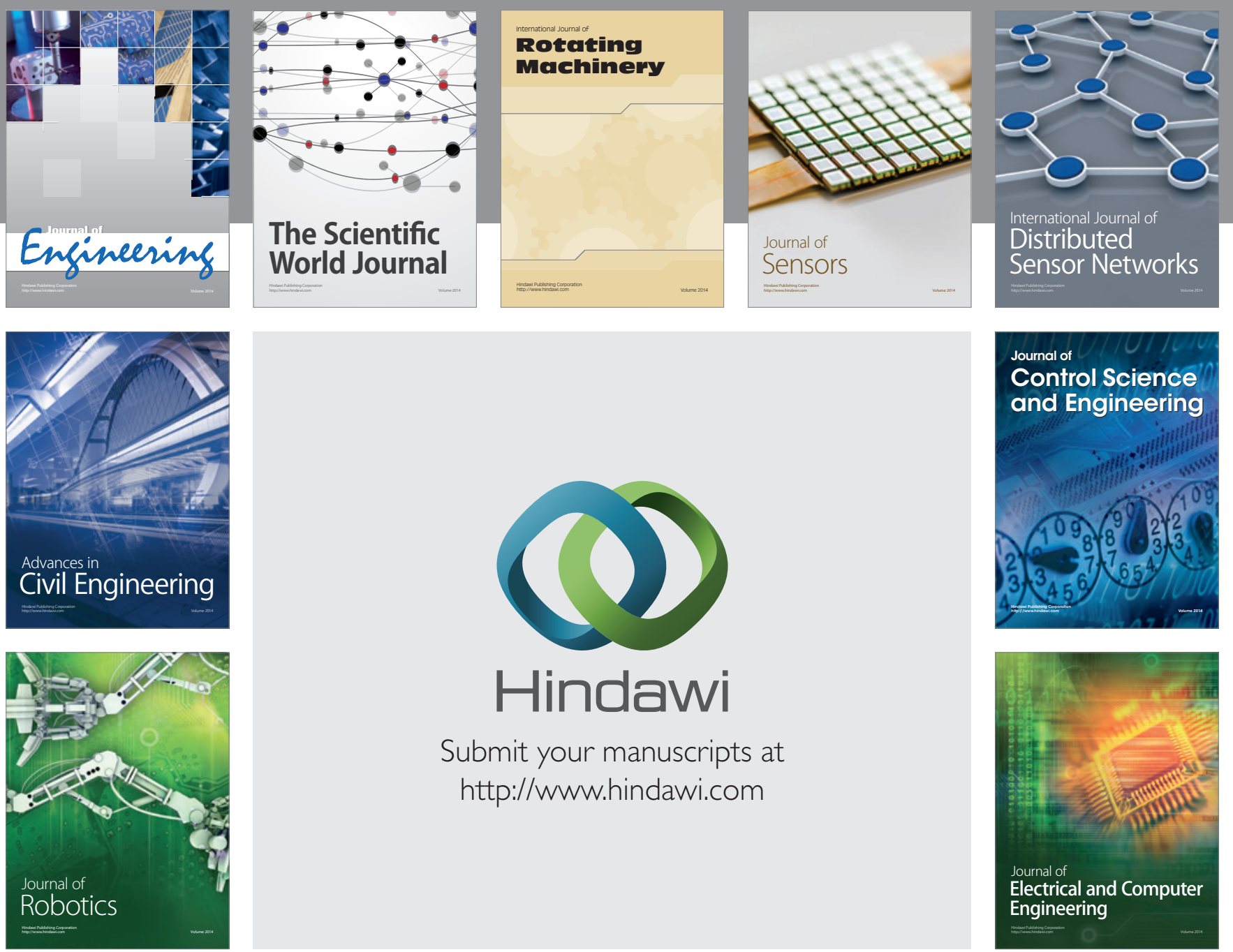

Submit your manuscripts at

http://www.hindawi.com
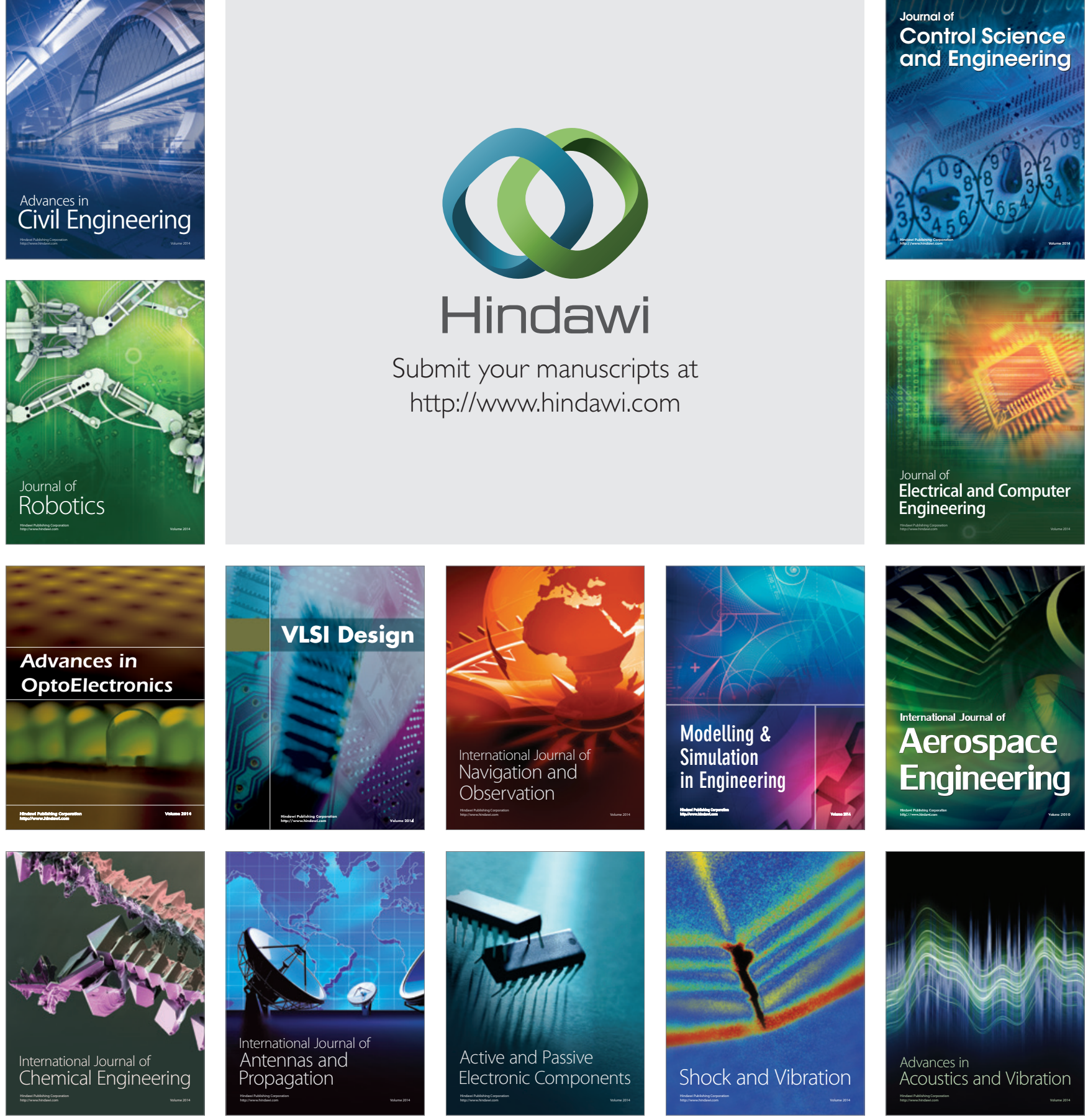